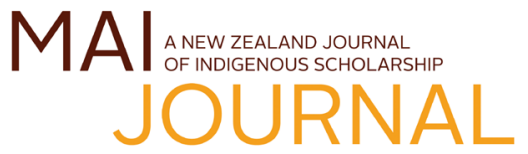

DOI: 10.20507/MAIJournal.2019.8.3.4

\title{
TE RANGIWHĀWHĀ O NGĀ ATUA MĀORI
}

\author{
Dr Dean P. S. Mabuta*
}

\section{He whakarāpopoto}

He kipakipa mutunga kore kua oho ake i te whatumanawa o te tangata kia mōhio anō ia ki ngā taonga tuku iho a ōna mātua tūpuna. E kitea ana i te hiakai o te iwi Māori whānui ki ngā kōrero mō Matariki, te maramataka Māori, te whakatere waka, te whakaora tikanga, me te tupu matomato rawa o ngā kaupapa whakarauora reo. Nā, ko te aronga nui o tēnei tuhinga he kuhu atu ki ngā kōrero tuku iho e pā ana ki ò tātou atua Māori. Inā rā, ko te matapae i tōna whai tikanga nui ki te whanaketanga o te tuakiri Māori, ka tahi. Ka rua, he whakatakoto tauira o tā te tangata Māori whakahāngai i ērā kōrero atua ki ōna horopaki hou o tēnei ao hurihuri. Āe rānei, ka whai take i tua atu o Rautaki, o Mahere?

\section{He kupu matua}

tikanga Māori, mātauranga Māori, kōrero tuku iho, te ao Māori

\section{Kupu whakataki}

Ko tō te Māori hononga ki ōna atua me ōna taiao te huinga o ngā muka ahurea, muka hìtori, muka tikanga, muka whakapono, muka akoranga, e kiia ai ia he Māori. He tirohanga whānui ki te ao Māori, kua mārō mai i ngā tūhonohonotanga i waenga i ngā mea katoa. Ka mutu, tārake ana te kite, ko tā te ao Pākehā whakamātau ki te wetewete, ki te whakamārama i te ao o te Māori, he ngoikore, kāore e tino mau ōna rangiwhāwhātanga, ka noho whāiti mai ki ngā kupu pēnei i te 'myth'. Waihoki, kāore ēnei momo kupu a te Pākehā e kapo mārika ana i ngā aweawe nui o Ngāi Tuawhakarere mā ki ngā mātauranga, me ngā kōrero tuku iho kua noho hei ture mō te iwi Māori ā mohoa noa nei. Heoi anō, hei tāpiri tonu ki ēnei whakaaro, ka mutu, me pono te kōrero ki a tātou anō, he māmā te tahuri ki ēnei kupu pēnei i te myth, i a tātou e kōrero noa ana me ngā hoa, e pahupahu ana. Nā, ki ōku nei whakaaro, i êtehi wā, ki te rongo te tangata, ana te tangata tauiwi i te kupu myth, ka āpiti ki runga i taua kupu ko ngā āhuatanga rūkahu, mana kore, arā, he kōrero i hangā noa hei whakangahau i te iwi. Mōku tonu, he pānga whakatoimaha tō ènei whakaaro ki runga i te tangata Māori (pēnei i te kaituhi nei) e hiahia ana ki te whakaatu i te whai mana o ngā kōrero tuku iho a te Māori ki te ao tauiwi. Tērā tērā horopaki. Nā, he aha tonu rā te raru nui? Ko te kupu, ko tā te tangata titiro ki te tikanga o taua kupu? Koinei pea tētehi uho nui o te whānuitanga o tēnei tuhinga, he whakatakoto, he whakaatu rā i te aronga o ngā whakaaro o te tangata ki tētehi āhuatanga kua roa e pūmau ana ki tōna ahurea, ka heke te wā ka huri te aronga, ka rerekē hoki te tirohanga a te whakatupuranga hou ki te ahurea, ki ōna wāhanga katoa, hītori, tikanga, kōrero tuku iho.

Nō reira, ko te mahi a te tuhinga nei he whakatakoto i êtehi o ngā kitenga tuatahi kua puta i aku rangahau e aro ana ki te mātauranga e pā ana ki o tātou atua, ā, ka arotakengia tā te tangata o nāianei whakahāngai i ēnei kōrero tuku iho ki ngā horopaki o te ao hurihuri nei. Ka whai wāhi atu ki te hoki whakamua ki ngā kōrero mō te orokotīmatanga mai o te ao, hei whakawhenua i a tātou, à, ka titiro ki ngā tauira whakaora kōrero atua i ēnei rangi. Haere nei te wā, e hāngai pū tonu ana ètehi o aua pou tikanga i takea mai i ngā atua, ki 
te tuakiri o te tangata Māori o ēnei rā. Nō reira he aha hoki ngā hononga hou kua waihangatia i a tātou e ngana nei ki te whakaora anō i ēnei taonga tuku iho.

Kia tōaitia te kōrero, ko te whanaungatanga i waenga i te Māori me tōna taiao, he ture ārahi i te raupapa, $\mathrm{i}$ te nahanaha o te mātauranga taketake o Aotearoa. E ai ki a Durie (2004), kāore e taea te Māori me tōna taiao te whakawehe;

While indigenous knowledge is often valued because of its traditional qualities, a creative and inventive capacity forms the core of an indigenous knowledge system. The perception of indigenous knowledge and culture as applicable only to the distant past misses the thrust for development that is part of the indigenous journey. Arising from the creative potential of indigenous knowledge is the prospect that it can be applied to modern times in parallel with other knowledge systems (wh. 1).

Mōku tonu, me taku pōtae rangahau Māori, ko tāku tirohanga he tirohanga kua tāraia ki ngā kōrero tuku iho nō roto mai o te iwi o Waikato. Waihoki, kei te pūtake o ōku whakaaro ko te whakapono o te tangata ki ōna atua, me kī, koinei te tino uho o taku rākau e māhuri ake nei. I te mutunga iho, e whakatairanga ana i te mana motuhake o tōku iwi Māori whānui, taku mana à-iwi taketake hoki, i te mea he rangahau tēnei ka hāngai pū ki taku whanaketanga. Mei kore ake ngā kupu a Te Rangihau (1974):

My being Maori is absolutely dependent on my history as a Tuhoe person ... Each tribe has its own history. And it's not a history that can be shared among others. How can I share with the history of Ngati Porou, of Te Arawa, of Waikato? Because I am not of those people. I am a Tuhoe person and all I can share in is Tuhoe history (wh. 232).

Kia hoki anō ki ngā kōrero a Durie, me te kī atu, koinei anō tētehi āhuatanga e whāia ana i tēnei tuhinga roa, he whakaatu i te hunga e ngana ana ki te whakahāngai i ō rātou mōhiotanga taketake ki ngā horopaki hou kua toko ake i te ao hurihuri nei. Ko tēnei hunga e whai ana i tā Durie kōrero, arā, kei te pūtake o te mātauranga ko te āheinga o te tangata ki te whakawhanake $\mathrm{i}$ roto $\mathrm{i}$ ana àhuatanga katoa. Ehake i te mea he toka tē taea te whao, engari he taonga ka whaowhina e ngā toki o tēnā whakatupuranga, o tēnā whakatupuranga. Nō reira, ko te pitomata e kitea ana me he waipuna tē mimiti, he koropupū mutunga kore. Kia hoki mai nei ki ngā kaupapa o te tuhinga nei, he titiro, ahakoa te aha-pai tū, pai hinga-e taea ana e te mātauranga Māori te whakakōrero i tō tātou ao hou e tūroa nei, e hurihuri nei.

Nō reira, tēnei ka mihi ki ngā taonga tuku iho o tōku ake kāinga. I tāraingia taku hoe ki ērā tikanga i whāngaihia mai nei ki ahau, à, ko taku rā i whatua mai ki ngā wheako o èrā i para tuatahi i tēnei huarahi o te whare wānanga, nā konā te pai o te matangirua o te waka ki ōna ūanga. Nā, tēnei te tuku i taku waka kia tere i te wai o Wānanga, o Mātauranga, me kore noa te kaipānui e whai i tētehi paku māramatanga, tōkihi!

\section{Te orokohanga o te ao-Tainui waka Te tīmatanga}

Kua hau ngā rongo o te kōrero mō Ranginui-etū-iho-nei rāua ko Papa-tūā-nuku (Rangi rāua ko Papa), tae atu hoki ki ā rāua tini uri atua, e noho nei hei whare tuakiri mō te iwi Māori. Ka ngana au ki te whakarāpopoto mai i ngā kōrero mō tēnei hunga, engari ka whāia ngā kōrero i takea mai ai i Tainui waka, ka mutu, ko ēnei kōrero i mātua tiki atu i ngā rangahau a Pei Te Hurinui. Inā te rētōtanga o ngā kōrero tuku iho nō roto mai o Tainui whānui e whakamārama ana i te ao atua, ka mutu, koia tērā te huarahi kāore anō kia takahia. Ki konei hoki kitea ai ngētehi kōrero mō Io, ka mutu koinei ngā kōrero tuatahi kua kitea e au e kōrero ana mō Io ki roto o Tainui, heoi, inā te nui o ngā kōrero mōna (Reed, 2004; PercySmith, 1913), à, puta ai te kōrero i ètehi wā, he atua $\mathrm{i}$ āpitiria nō te taenga mai o te Karaitiana. I ēnei tuhinga, kāore a Te Hurinui e wewete i ēnei kōrero, ko tāna noa he àta whakatakoto i ngā kōrero i tukuna ki a ia e ngā tohunga o tētehi o ngā whare wānanga o Tainui.

E ai ki a Jones (2010, 2013), ko te tīmatanga mai o ngā mea katoa i hua mai i roto i a Io, me tana whanaketanga mai i Te Kore. E waru ōna wā, ōna whakapaparanga o Te Kore, ā, ko te tuawaru o ēnei whakapaparanga ko te pūāwaitanga mai o te mana orokohanga, o Io. Koinei te tīmatanga i mua noa atu i te whānautanga mai o Rangi rāua ko Papa. Waihoki, i te whanake haeretanga o ēnei wā e waru, tērā ngā ira orokohanga i whakakotahi mai ki roto pū i a Io, ko te ira tāne, ko Hani, ko te ira wahine, ko Puna, (Jones, 2010). I tēnei wā ko tā rāua mahi he rere haere i Ngā Rangi Tūhāhā ki te kimi wāhi e taea ai e rāua te piri. Ka mutu, ahakoa ka whakawhiti tō rāua ara ki Te Ahurewa Tapu o Io, nā Te Tumu i wehe tonu ai rāua, tē piri.

Ki tua o te pūāwaitanga o Io, ka puta i a ia ko ngā whetū, ā, nā ngā whetū ka puta ko ngā tātai whakapapa e rua, ko te tātai o Te Pō me te tātai o Te Ao. Ka whakatinana mai ēnei tātai i a Tau 
Ana Te Marama (Te Pō) rāua ko Te Rā (Te Ao). He roa te whakapapa nei, ka heke haere i ngā pō, ka heke haere i ngā ao, ā, kia puta rawa i ngā tātai whakaheke o Te Po, ko Papa-tūā-nuku, ā, i ngā tātai whakaheke o Te Ao, ko Ranginui-e-tū-ihonei (Jones, 2010).

Nō te putanga rā anō o Rangi rāua ko Papa, ka whai wāhi a Hani rāua ko Puna ki te piri, ā, i te uma o Papa, ka piri tahi ai rāua. Mai i tēnei hononga ka puta tuatahi ko ngā ika me ngā mea katoa e ora ana i te moana, ko èrā ika me ngā mea ora o ngā wai Māori, tae atu ki ngā mea katoa e ora ana i runga i te whenua (Jones, 2010). Hei tā Jones (2010), katoa ēnei mea orokohanga ka noho hei kai whakapiki i te mauri ora o ngā uwha me ngā tame ka tupu i te ao whānui.

I mua i te whānautanga mai o ngā tamariki a Rangi rāua ko Papa, kāore rāua e piri ana, engari i te moe wehe kē rāua. Nā te huinga atu o Hani rāua ko Puna i piri ai a Rangi rāua ko Papa. Ki roto anō i a Jones (2010), ka puta te kōrero nā Hani a Rangi i whakaoho, ā, i taua wā tonu ka whānau tōkaikore mai êtehi uri o Rangi, tekau mā whitu katoa ngāna uri, ko te tamaiti whakamutunga a Rangi ko Tānepukurua. I whiwhi ia i tēnei ingoa i runga anō i te putanga tōkai-kore mai o ngā uri a Rangi, ahakoa he tāne (Jones, 2010). I te whānautanga mai o Tānepukurua ka puta mai te puku tuarua o Rangi, ka whānau pī mai a Tānepukurua. I tēnei wā, ka taka te whenua ki runga i a Papa, nā wai rā i ohorere a Papa ka whānau tōkai-kore mai hoki he uri, tekau mā whā katoa rātou.

I te wā e whānau uri ana a Papa, koinei te wā i hangā te āhua o te whenua, arā, ka nukunuku te whenua, ka nekeneke te whenua, ka hiki ngā maunga, ka wehewehe ngā wai katoa. I muri i te whānau mai o ngā uri katoa o Papa, ka whānau pī anō a Rangi kia rua anō ngā uri, à, ka taka ngā tokorua nei ki runga i a Papa, ko ō rāua ingoa ko Rimurehia rāua ko Te Awekōpara. Mai i a Rimurehia ka puta ko ngā otaota katoa o te moana me ngā roto. Mai i a Te Awekōpara ko ngā huruhuru manu katoa. Ka taka ngā tamaiti kokoti tau e rua nei ki a Papa, ka mutu, he tangi pāorooro i rangona i ngā tōpito katoa o ngā rangi tūhā, mea rawa ake, kua oho mai a Aituā, ā, ka mau atu ia ki te pou kōwhatu, ki Te Tumu, e wehewehe nei i a Rangi rāua ko Papa. Mai i taua wā, i tapaina a Aituā ko Tūtangatakino. Heoi, ka puta mai a Io, ka karanga me tētehi wāhanga o tōna mana, o tōna wehi ki a Tūtangatakino kia haere. Kotahi tonu atu a Aituā, waihoki, ka hinga mārika Te Tumu, ka rere a Rangi ki a Papa, ā, ka piri mārika rāua. I tēnei piringa o Rangi rāua ko Papa ka puta ko ngā atua rangatira e mōhiotia whānuitia ana e te iwi
Māori: Ko Rongo rātou ko Tānemahuta (Tāne), ko Haumiatikitiki (Haumia), ko Tāwhirimātea (Tāwhiri), ko Tangaroa, ko Tūmatauenga (Tū), ko Rūaimoko (Rū), ko Kahukura (Jones, 2010).

\section{Ranginui rāua ko Papatūānuku}

Ka huri atu ināianei ki te wehenga o Rangi rāua ko Papa. Nā te mea he tino kōpā te noho o Rangi rāua ko Papa, ka hiahia ngā tamariki atua nei ki te puta ki te ao mārama, ā, i pupū ake te whakaaro me whakawehe rātou i o rātou mātua kia wehe noa. I roto i ngā kōrero nei, tokoono ngā atua i noho ki te wānanga me aha atu rātou ki ō rātou mātua; ko Tāne rātou ko Tāwhiri, ko Tangaroa, ko Tū, ko Rongo, ko Haumia. E ai ki a Tainui, e rima atu anō ngā ingoa o Tūmatauenga, arā, ko Tūmatawhāiti, ko Tūkariri, ko Tūkanguha, ko Tūkaitaua, ko Tūmatateueue (Jones, 1959). I roto i ngā kōwhiringa, nā Tū te kōrero me patu rātou i a Rangi rāua ko Papa kia mate rawa, engari kāore a Tāne i whakaae. Ki a Tāne, me whakawehe noa rāua, i runga i tērā i ngana ia atua ki te whakawehe i a Rangi rāua ko Papa. I te tuatahi, i ngana ko Haumia rāua ko Rongo, ahakoa tō rāua kaha, tē taea e rāua te whakawehe ō rāua mātua. Ka ngana hoki ko Tū rāua ko Tangaroa, pērā anō hoki rāua, tè taea te whakawehe a Rangi rāua ko Papa. Kāore a Tāwhiri i whakaae kia wehea ngōna mātua nā tōna aroha mō rāua tahi. Nō reira, kāore ia i ngana ki te mahi pērā i ngōna tuākana, tēina hoki. Kāore i taea e ngā atua te whakawehe a Rangi rāua ko Papa. Ka tū ake ko Tāne, engari i rerekē te āhua o tāna mahi, i takoto ko tōna tuarā ki a Papa, ā, ko ngōna waewae ki tōna matua, ki a Rangi, ā, nā ngōna waewae i taea ai e Tāne tōna matua te pana ki runga ake kia uru mai ko te ao mārama.

Nā Tāne a Rangi rāua ko Papa i whakawehe, engari kāore tonu a Tāwhiri i whakaae ki tēnei mahi. Waihoki, ehake $\mathrm{i}$ te mea $\mathrm{i}$ te whakawehe noa ngā atua nei i ō rātou mātua, engari rā ko te mutunga kē mai nei o te pāmamae, me he aituā ki runga i te ao. Nā tēnei mamae nui i whakaaro ai a Tāwhiri ki te kimi utu mō te hara nui nei (White, 1890). Nō konei ka tīmata te pakanga i waenga i ngā tamariki a Rangi rāua ko Papa, te pakanga a ngā atua.

\section{Te Paerangi}

Nā te wehenga o Rangi rāua ko Papa i puta mai ai te ao mārama, ā, ka noho tēnā atua ki tōna wao, tēnā atua ki tōna takiwā, tēnā atua ki tōna kauwhanga. Ko Tāne te atua o ngā ngāherehere me ngā kararehe katoa e noho ana ki reira, arā, ngā manu tae atu ki ngā ngāngara. Ko Tangaroa te atua o ngā moana me ngā ika katoa. Heoi anō, 
a Tāwhiri, nā tōna tino aroha mō ngōna mātua i piki ake ia ki ngā rangi tūhāhā, ki te taha o tōna matua, o Rangi, ki reira noho ai hei hoa mōna, $\bar{a}$, i tū ko ia te atua o ngā hau. Nā te kaha whawhai me te riri o Tū kua mōhiotia ia i ngēnei rā ko te atua o te pakanga me te tangata (White, 1890). I tēnei wā, ka whakaaro a Tāwhiri ki te kimi utu mō ngā hara o ngōna tuākana, tēina hoki. Nō reira, i heke iho a Tāwhiri, i whawhai tahi ia me tōna whānau. Ka haere ia ki roto i ngā wao a Tāne me ngōna hau kaha, ki reira pupuhi kino ai i ngā rākau a Tāne, me te whiu haere i ngā kāinga o ngā manu me ngā kararehe katoa o te ngahere. I muri ake i rere ia ki ngā moana, ki te kāinga o Tangaroa, ki konei whakapupuke ai te moana kia ngarungaru. Ko ngā ngaru nei i piki ki ngā rangi, ko ngā ika katoa o te moana i wehi, à, nāwai i wehi ka mauri rere. I waenganui i te pakanga nei a Ika-tere rāua ko Tū-te-wehiwehi e oma mataku nei, ko Ika-tere te matua o ngā ika katoa, ā, ko Tū-te-wehiwehi te matua o ngā ngāngara. Nā te kaha o Tāwhiri i amuamu tahi ai rāua, i hiahia a Ika-tere ki te noho ki te moana huna ai. Engari i rerekē tō Tù-te-wehiwehi whakaaro, i hiahia kē ia ki te oma ki runga ki te whenua. I te mutunga ake, i wehe rāua me tā rāua karanga anō ki a rāua, ka noho rāua hei kai mā te tangata.

I muri ake i te pakanga i waenga i a Tāwhiri rāua ko Tangaroa, i tū mai ko Tū ki te whawhai ki a Tāwhiri. O ngā atua katoa, ko ia anahe i taea te karo te kaha o Tāwhiri, kāore i taea e Tāwhiri a Tū te patu, engari kāore hoki a Tū i toa i a Tāwhiri. I te mutunga ake, he ōrite te kaha o Tāwhiri rāua ko Tū. Nō reira, i tau te puehu, i hoki a Tāwhiri ki te taha o tōna matua, o Rangi. Heoi anō, kāore ngā riri i mutu i reira, nā te mea kāore i taea e Tāne rātou ko Tangaroa, ko Haumia, ko Rongo te karo te riri o Tāwhiri, i kimi utu a Tū i tō rātou koretake. I te tuatahi, i haere a Tū ki te hopu i ngā manu me ngā kararehe o te wao a Tāne hei kai māna, ā, ki ngā rākau o te ngahere i hangaia e Tū ngā taputapu mā te tangata. I muri ake i hanga kupenga a Tū ki te hopu i ngā ika o te moana hei kai hoki māna. I mua i te pakanga ki a Tāwhiri, i mōhio a Tū kua huna a Haumia rāua ko Rongo i te taha o tō rātou whaea, o Papa. Nā tēnei i kimi a Tū i a rāua, ā, nā te karakia i huri a Haumia rāua ko Rongo hei kai, ko Haumia te aruhe, ko Rongo te kūmara. Nā tēnei i noho ko Haumia te atua o te aruhe, arā, ko ngā kai katoa ka tupu ki roto i te ngahere. Ko Rongo te atua o ngā kai ka whakatupua ki roto i te māra (White, 1890).

Koinei ngā kōrero ka noho hei tūāpapa mōku i roto i aku mahi o ia rā, tae atu ki ōku hiahia mō aku mahi rangahau. Tāpiri atu ki ēnei kōrero, i roto i aku wānanga, ki tāku nei titiro, ko ngā kōrero mō Hani rāua ko Puna, he kōrero motuhake nō roto mai o Tainui. Tae atu hoki ki ngā kōrero tīmatatanga mō Hani rāua ko Puna me Rangi rāua ko Papa. Waihoki, ko ngā kōrero mō Io e tautohetohengia ana e te ao rangahau i runga i te whakaaro he āpitihanga a Io ki ngā kōrero atua Māori nā ngā karaipiture, nā ngā Hāhi Tauiwi. Mōku tonu, e whakapono ana au ki a Io me te whakaaro he mea kōrero e ngā tuhinga a Pei Te Hurinui Jones, ka tahi. Ka rua, e kōrerotia ana ki roto i ērā atu kohinga tuhinga Māori, pēnei i a Te Whatahoro mā, i a Te Mātorohanga mā. Ka toru, e mōhiotia ana a Io ki ètehi iwi o Te Moana-nui-a-Kiwa, pēnei i a Hawai'i, nō reira, tērā ōku whakaaro e whakapono ana, he mana nō tua whakarere a Io, ko te raru kē, ko tā te Pākehā whakahāngai i tō rātou atua ki ō te Māori atua. Heoi anō rā, he kaupapa anō tēnei hei tuhi māku, ā, me mihi ka tika ki ēnei taonga kōrero i whakaemingia e Pei Te Hurinui hei taonga tūhurahura mā tātou o ēnei rā, kia kitea ai ngā rerekētanga ki ērā atu iwi, arā, te whakatinanatanga o te mana motuhake $\mathrm{i}$ kōrerotia e Te Rangihau (1975).

\section{Te ao hurihuri, te ao o nāianei}

Mehemea ko ngā kōrero kua whārikihia ki runga ake nei te tūāpapa o te ao Māori, ā, he kōrero tuku iho ēnei nō tua whakarere i mārama pū ki ngā tūpuna Māori, ka toko ake te pātai, āe rānei e taea ana ēnei kōrero tuku iho te whakahāngai ki te ao Māori o nāianei, mehemea kāore e mārama tōna tino tikanga ki ōna uri o nāianei? Ki ōku nei whakaaro, e ora ai te wairua Māori, te wairua taketake o te uri Māori, me whakamātau. Heoi anō rā, me tika tā tātou raweke i ēnei kōrero i à tātou e kimikimi haere ana, e whakawhanake haere ana i tō tātou tuakiri. Me āta wānanga, ka tika, tā te tangata whakahāngai i ēnei kōrero atua ki te oranga o te tangata i tēnei ao hurihuri.

Nā, i a au e tīmata nei ki te āta wetewete i ngā kōrero atua, me te mātai hoki i ngā tauira e kitea ana i ēnei rangi, arā, ērā hunga kua tīmata ki te hauhake i ngā kōrero tuku iho ka whakahāngai ai ki ngā horopaki o ēnei rā, e toru pea ngā tino tāhū matua e kitea ana:

1. Ko te whakarauora i ngā tikanga tuku iho kua ngaro haere, kua roa e moe ana, kia ora anō ai i ēnei rā. Hei tauira, ko te whakatere waka, ngā mahi toi, te whaikōrero, te karanga, ngā kōrero mō te whānau mārama, te maramataka Māori, te karakia.

2. Te ao hauora, hākinakina, kai ora, tinana ora, èrā tū kaupapa. 
3. Te ao matihiko, arā, te ipurangi, inā koa ngā momo pāpāho hou, pēnei i te īpae Pukamata, TiriAta, ngā rangitaki, ngā kēmu ātea.

Nō reira, e tika ana kia huaina ètehi tauira o ēnei tāhū kaupapa e toru nei, me kore noa e whānau mai he paku māramatanga ki a tātou e kimikimi noa nei, e kỉia ai tātou he Māori.

\section{Te whakarauora tikanga tuku iho-Matariki/ whakatere waka}

Inā te nui o ngā tauira whakaora kōrero tuku iho, whakahaumaru tikanga tuku iho, ā, mōku tonu, koinei te whare mātauranga e pai ana ki ahau, hei nōhanga mō taku rangahau. Kua roa kē te ao Māori, te ao rangahau, e tūhurahura ana i ngā taonga a ō tātou tūpuna mātua hei whakaoranga wairua, hei whakapakari tuakiri. Haere nei te wā, kua oho mārika te whatumanawa o te hapori Māori, o te iwi Māori whānui ki ngā kōrero tuku iho e hāngai ana ki te whānau mārama, te maramataka Māori, me te whakatere waka. Hei tauira, pēnā ka tirohia a Matariki, tērā kāhui whetū e kìia ana ko Pleiades ki te ao tauiwi (Matamua, 2017; Best, 1922), te tohu nui o te tau hou Māori, kua roa ngā kōrero mōna e moe ana. Āe, tērā te kōrero, i mōhio ètehi Māori ko Matariki te tohu o te tau hou Māori, engari nō ēnei tau tata nei i whakaaraarangia ai, i whakaohoohongia anō ai tētehi momo tikanga hei whakanui i a ia.

Mōku tonu, me mātua kī atu, e pēnei ana te aranga mai o ngā tikanga tuku iho i runga anō i te piki haere o te mana o ngā kōrero tuku iho, o ngā mātauranga nō tūāukiuki, ā, me te hiakai o te iwi Māori whānui ki ēnei taonga tuku iho. Hei takoha i tētehi tauira, nōku e taitamariki ana, e pono ana te kōrero, i whāngaihia tuatahitia ngā kōrero mō Rangi rāua ko Papa, te pakanga o ngā atua, ā tōna wā, kua rongo kōrero mō Matariki. Ahakoa tēnei, i a au e tū pakeke nei, ka pūrangiaho, i te karetai noa o te moana au e kauhoe ana, ka mutu kāore i paku mārama ki tōna hōhonutanga. Kāore i whakanui i a Matariki pēnei i a tātou i ēnei rangi tata nei, ā, ehake i te mea he kaupapa i rite tonu te whakarite ki te wātaka o te tangata. I ènei rā, māku e kī atu, kua panoni mārika tēnei āhuatanga.

Me te aha anō, me taku tirohanga Māori, nā te angitū o ngā kaupapa pēnei i a Hokule'a, me te whakarauoratanga o ngā tikanga whakatere waka pōkai moana i tīmata i ngā tau '70, i mauri oho anō te whakaaro nui ki ō te Māori mōhio, ki ā te Māori tikanga, ki ō te Māori mātauranga tuku iho e hāngai ana ki ēnei horopaki. Ko Te Toki Waka Hourua tētehi o ngā rangapū whakatere waka Māori, i raro i te maru o Hoturoa Kerr o
Tainui. Ko te kaupapa matua o te kāhui nei he whakarauora, he whāngai hoki i ngā kōrero tuku iho mō te whakatere waka, me te whakatairanga i tōna oranga tonutanga ki waenga o te iwi (Te Toki Waka Hourua Voyaging Trust, 2018). He rite tonu tā Te Toki Waka Hourua whakahaere hōtaka ako i ngā pūkenga whakatere waka. Ko Haunui te ingoa o tō rātou waka hourua rangatira, ā, tere ai ia ki ngā tōpito o Te Moana-nui-a-Kiwa.

Haere nei te wā, kua kitea te pikinga ora o te mātauranga mō te whānau mārama, ā, nō ngā tau e rima kua pahure ake nei kua papā te whaititiri, kua hikohiko te uira i te ohonga mārikatanga o te iwi Māori ki tēnei kaupapa. Mātua rā ko Tākuta Rangi Mātāmua, ko ia kei te aroākapa o tēnei ohonga mōhiotanga, me mihi ka tika ana hekenga werawera mōna i ruku rētō ki te taonga tuku iho i waiho mai e tōna matua koroua, he kōrero tuku iho nō te ao tawhito, kua hauhakengia hei oranga mō te iwi Māori o te ao hurihuri. Ko tōna pukapuka, Matariki: Te Whetū Tapu o te Tau (2017), te putanga tuatahi o ana rangahau ki te motu, ā, hei hāpai i ēnei kōrero, kua rite tonu tana kauwhau ki runga ipurangi, tana whakatakoto kōrero mā runga Pukamata, tana kawe hoki i te kaupapa ki ngā rohe katoa o te motu, i runga anō i tōna ngākaunui ki te oranga tonutanga o ēnei kōrero tuku iho ki roto pū i te iwi Māori whānui. Mō te tūpono ka mahue i ahau ētehi kupu mihi anō, kātahi nā te tauira o te rangahau, i oho ai a Tiki-apoa i te whatumanawa o te kaituhi nei ki te whai i tōna ake huarahi rangahau mō ngā atua o te ao Māori.

\section{Te Hauora me te ao hākinakina}

Ko te ao hauora tētehi o ngā tino horopaki, tētehi o ngā tino kaupapa kua whai pānga nui ki te hauhakenga mātauranga e pā ana ki ngā atua Māori hei ārahi i ā rātou mahi. I tīmata pea i a Tā Durie, ki te pou tarāwaho e kīia nei ko Te Whare Tapawhā (Durie, 1998). Ina rā, ko te pānga nui o te taha wairua ki roto i tēnei momo pou tarāwaho, hei hapahāpai i ngā mahere hauora o te motu, ka mutu, i ngā mahere hauora e hāngai pū ana ki te tangata Māori.

Waihoki, kua kitea tēnei momo tauira i roto i ètehi atu āhuatanga o te ao hauora. Tèrā te tauira i puta i a Tākuta Ihirangi Heke, me tōna mahere hauora, a Atua Matua (Heke, 2017)—e toro atu ana ki ngā kōrero tuku iho mō ngā atua Māori hei ārahi i tā te tangata whakapakari tinana. Āpiti atu ki tēnei, kua roa a Tākuta Heke e tōmene ana i ngā taunakitanga o te mana tūhonohono o te whakapapa hei aho tuitui i ngā whakaaro kei te mahere nei o Atua Matua. Hei tāna, ehake i te mea, 
he herenga tangata anahe te mahi a te whakapapa, engari ia he pūnaha whakarite, whakatakoto i te ao o te Māori, e mārama ai ia ki ōna hononga katoa (Heke, 2017). Mōku tonu, koinei tētehi tauira pai, e whakaatu ana i tā te tangata hauhake i ngā kōrero atua, ka whakahāngai ai ki tētehi mahere hou hei whakatauira i tēnei horopaki hou, arā, ko ngā mahi a Tāne ki te whakawehe i ōna mātua, ko tōna tuarā ki tōna whaea, ko ōna waewae ki tōna matua, ka pana whakarunga me ona waewae, ko te 'leg press' tuatahi-nā, koinei te tūāpapa o ngā rangahau a Heke.

Mōe ētehi tau, tērā tētehi rangitaki rongonui, ko 'Whakapapa Fridays', nā Te Miri Rangi, i whakatūria hei huarahi whāngai mātauranga Māori e tautoko ana i te hauora o te tangata. Nō te kura huna kotahi a Rangi rāua ko Heke, he wetewete, he wānanga, he mātai i ngā kōrero e pā ana ki ngā atua Māori ki te kimi tauira, ki te kimi āwhina mō te oranga pai o te iwi Māori.

Nō ēnei tau tata nei, tērā tētehi kaupapa, ko Hawaiki Toa tōna īngoa, i whakatūria e Beez Te Waati, hei huarahi tūhonohono i te tangata Māori ki ōna atua mā roto mai i êtehi wero whakapakari, whakangungu tinana. He hōtaka whakapakari tinana, i tōna mutunga iho, he whakataetae kori tinana kua waihangatia i raro i ētehi kōrero atua.

\section{Te ao matihiko-te îPae, te kēmu ātea me te kiriata}

Hei whakaoti atu i taku whakatakoto tauira, e tika ana kia tirohia te ao matihiko. I te ao Māori e āta kuhu atu ana ki ngā riu matihiko o tēnei ao hurihuri, ia rā, ia rā, e kawea ana e tātou tō tātou ahurea, à tātou tikanga, tae atu anō hoki ki ō tātou atua, ki ēnei rohe hou. Nāwai nāwai, kua tīmata ēnei wāhi hou ki te whakaaweawe i tā te Māori toro atu ki te riu o ōna atua, ā, ka whakaawe hoki i tā te Māori whakamārama i tōna ao ki ērā atu iwi (Ka'ai-Mahuta \& Mahuta, 2017). Inā te mahi a te tauira matihiko e whakamahia ana ngā kōrero atua, mai i ngā pūmanawa tautono hei waka tiaki i ēnei kōrero, hei waka tuku hoki i ēnei kōrero; ki runga i a TiriAta, tērā ètehi whitiata whakarākei pani mata e peita ana i ngā mata atua wāhine ki te mata o te tangata; kei te wānangatia te ao atua ki runga i a Pukamata; katoa ēnei āhuatanga e whakahaerengia ana i tēnei wā tonu nei.

Ko tētehi āhuatanga nui e kitea ana e au, i roto i ngā tau tekau kua pahemo, kua kaha ake tā te tangata (tauiwi mai, taketake mai) toro atu ki ngā kōrero atua hei kaupapa mō ngā kēmu ātea me ngā kiriata. Nō ngā tau '90 te kēmu, The Mark of Kri, ā, he Māori te āhua o te tuahangata, he moko tōna, he taiaha tētehi o ana rākau pakanga. I ētehi wā ka kōrero ia ki tētehi "wairua" o te ngahere hei ārahi i a ia. Nā, ahakoa te pārekareka ki te tākaro, katoa ngā āhuatanga Māori o te kēmu, kāore i whakamahia ki tōna aronga tika, arā, te aronga e ai ki te ao Māori (Mahuta, 2013). In arā ko te tuatahi tēēā, ā, peka mai ki ēnei tau tonu, kua nui noa atu ngā wā i kitea ai te ahurea Māori ki te paepae o te ao hurihuri. Nō te tau 2016, i puta ai te kiriata Moana, nā Disney, e kōrero ana te pūrākau mō tētehi puhi ariki i reia e ia te moana ki te kimi i a Māui, māna e whakaora ngā motu o te moana nui. I te mutunga iho, i āhua rite te whakaaro ki tō The Mark of Kri, he pārekareka rawa atu ā-kiriata, ā-pūrākau, engari ko ngā kōrero mō Māui, ko ōna kōrero tuku iho kāore i rite ki tā te Māori mōna i te mea i whenumi katoa tēnei "kawenga" o Māui kia hāngai ki ngā iwi whānui o Poronīhia. Kotahi noa iho tōna painga, arā, i whakaae a Disney kia whakamāoringia te kiriata nei, ā, i tērā, i kaha ake tō te iwi Māori pārekareka ki tēnei kiriata. Taihoa ake, mō tuhinga kē wetewetehia ai tēnei kaupapa! Ko te kupu matua o tēnei, ahakoa te hiahia o te ao Māori, e kaha whakamahia ana ō tātou kōrero tuku iho hei whakarākei i ngā "taonga tākaro" o ēnei rā.

\section{He kupu arohaehae}

Ki te tahuri au ki te arohaehae i ētehi o ngā whakaaro kua puta, kāore au e kī ana e hapa ana, e hē ana rānei ēnei tauira, engari ia me mātua wānanga tōna whakamahinga i te tuatahi. Nā te hauhake kōrero, kua whāiroiro te ao me ngā hinengaro o ō tātou mātua tūpuna, à, nā tēnei kua hiakai ake te Māori ki te rapu i ērā kura huna o tūāuriuri, o whāioio. Katoa ngā mātauranga Māori e hono ana ki ngā kōrero mō ngā atua mā roto mai o te whakapapa. Ko ngā whakapaparanga, ko ngā tūhonohonotanga i waenga i te iwi tangata me te taiao te tūāpapa mō te tuakiri o te Māori. Nō reira, he take tino nui mā tātou te iwi Māori whānui te āta whakaaro ki tēnei whanaungatanga ki ō tātou kōrero tuku iho, me tā tātou whakamahi i ēnei kōrero tuku iho hei tārai i te tuakiri Māori o ēnei rangi nei-e kīia tonutia ai he tuakiri Māori. Taihoa ake te whakamotuhake anō ki ngā kōrero tuku iho o tēnā waka, o tēnā iwi, o tēnā hapū, o tēnā whānau!

Ka toko ake te pātai, ka pēwhea tā tātou whakamana i te tū a ō tātou atua ki tō tātou oranga? Kei noho noa hei mahere, hei rautaki ārahi i à tātou mahi o ia rā, o ia rā, hei tikanga rangahau hoki mō ngā kaupapa huhua e whai pānga ana ki te ao Māori o nāianei. Kei whea tōna tūranga nui hei aho paihere i te wairua o te tangata? Ka puta anō te pātai i te waha, i a tātou e tū Māori nei, ka rongo 
rānei au i taku hono ki ōku atua? Nā, pēnā tātou ka hoki anō ki te tīmatanga, ki ngā kōrero a Durie (2004)—ko tētehi wāhanga nui o te mātauranga Māori, ko tōna āhei ki te hono ki te ao o te tangata e huri ana, e whanake ana-nō reira mehemea tātou e whakaae ana ki ēnei kōrero āna, me mutu rānei tā tātou hauhake kōrero tuku iho? Me mutu tā tātou whakahāngai ki ngā horopaki hou? Ko te āhua nei me kaupare atu ēnei tū whakaaro, me hoe tonu te waka, me kaha tonu tā tātou kimi i te oranga o te mātauranga ki ngā horopaki hou.

Ko tētehi o ngā tino akoranga o tēnei, o ngà kaupapa rangahau à haere ake nei, tārakerake ana te kitea kei tua o rangiwhāwhā te riu o ō tātou atua, à, e taurite ana te whānuitanga nei ki te mōhio o ō tātou mātua tūpuna, he pae tē kitea! Tērā tētehi kōrero i kitea e au i roto i aku rangahau i ngā tuhinga tawhito kei Te Uare Taoka o Hākena, te whare tiaki tuhinga tawhito ki Ōtepoti, ki ngā kāhui tuhinga a Edward Shortland. He whakamārama mō tētehi karakia hei whakaora i te wahine whāngai pēpi, à, kāore e rere ana te wai ū. Ko te whakarāpopototanga o te rautaki a te tohunga $\mathrm{i}$ haere atu ki te tiaki i te wahine nei, he takutaku, ka whakahau kia noho wehe a māmā rāua ko pēpi mō te wiki kotahi, ka whakakotahi mai anō kia karakiangia e te tohunga, ā, ki reira rere ai te wai ù. Mīharo katoa au ki tēnei tauira o te mana o ō tātou tūpuna, o ō tātou tohunga o te ao kōwhatu. Heoi anō rā, nāwai i mīharo, ka tumeke rawa atu i te kitenga atu o tētehi wāhanga o te karakia i whakamahia hei āwhina i te whaea nei, me te atua i karangahia hei mana āwhina, inā koa:

Tù-te-awhiawhi (Tūu-the-embracing)

Tù-te-panapana (Tū-the-pulsating-one)

Tū-te-pupuke (Tū-who-causes-the-watersto-well-up)

(MS-0002, wh. 46)

I whakamānawatia te mana atua o $\mathrm{T} \bar{u}$, te atua o te riri, o te pakanga. Kapakapa ana te manawa, i te mea, ohorere ana tēnei pia wānanga i te korenga o Rongo, i te korenga o Hine-te-iwaiwa, o wai ake rānei o ngā atua e kīia ana e ngā pukapuka, e ngā rangahau o ènei rā, ko ia pū te mana takutaku mō ngā tikanga wāhine. Ki konei tīmata ai te hinengaro ki te āta wāwāhi kia kuhu mai ko Whakaaro Hou, ko Aronga Hou.

Mōku tonu, ka noho tēnei taonga tuku iho hei toki wāwāhi toka, he tauira o te tino mōhio o ō tātou mātua tūpuna ki tō rātou ao, me ōna atua, i taea ai e rātou te whakarite tūhonohono tika i runga anō i te hiahia. Ki konei hoki kī atu ai au, e taea ana ngā mea katoa, ko te mea nui me mārama ki ō tātou hononga, me mōhio, me wānanga i mua, kei riro te Māori hei papa mō Kūware.

\section{Rārangi pukapuka}

Best, E. (1922). The Astronomical Knowledge of the Māori. Te Whanganui-a-Tara: Dominion Museum.

Durie, M. (1998). Whaiora: Maori health development. Tāmaki-makau-rau: Oxford University Press.

Heke, I. (2017). Mātauranga Māori and Systems Dynamics: Where old world collides with the new. He pepa i tango mai i www.toitangata.co.nz

Jones, P. T. H. (2010). King Pōtatau. An account of the Life of Pōtatan Te Wherowhero, the first Māori King. Te Whanganui-a-Tara: Huia Publishers \& The Polynesian Society.

Jones, P. T. H. (2013). He Tubi Mareikura. Ngā kōrero a te Māori mō te Hanganga mai o te Ao nō ngā Whare Wānanga o Tainui. Kirikiriroa: Aka \& Associates.

Ka'ai, T. M. et al. (2004). Ki Te Whaiao. An Introduction to Māori Culture and Society. Tāmaki-makau-rau: Pearson Education.

Ka'ai-Mahuta, R. T. A. \& Mahuta, D. (2017). Te Matarua o te Hangarau. MAI Journal 2017, 6(3). pp. 344-352. https://doi.org/10.20507/ MAIJournal.2017.6.3.9

Mahuta, D. P. S. (2013). Hinga atu a Tāne-rore ki te ipu o te rangi. Ki roto i a R. T. A. Ka 'ai-Mahuta, T. M. Ka 'ai, \& J. C. Moorfield (Eds), Kia ronaki: the Māori performing arts. (pp. 273-277). Tāmakimakau-rau: Pearson Education.

Matamua, R. (2017). Matariki: Te whetū tapu o te tau. Te Whanganui-a-Tara: Huia Publishers.

Rangihau, J. (1974). Being Maori. Ki roto i a M. King (Ed.), Te Ao Huriburi-The World Moves On. (pp. 221-233). Te Whanganui-a-Tara: Hicks Smith \& Sons Ltd.

Reed, A. W. (2004). Reed Book of Māori Mythology. Tāmaki-makau-rau: Reed Publishing.

Te Toki Waka Hourua Voyaging Trust. (2018). www. tetoki wakahourua.org

Tuaupiki, J. W. (2017). E Kore E Ngaro, He Takere Waka Nui: Te Mātauranga Whakatere Waka Me Ona Take Nunui. (Tuhinga Kairangi, Te Whare Wānanga o Waikato, Kirikiriroa, Aotearoa). Tango mai i https://hdl.handle.net/10289/11654

White, J. (1890). Tainui. Ki roto i a The Ancient History of the Maori, his Mythology and Traditions. (Vol. 6). Te Whanganui-a-Tara: Didsbury Government Printer.

\section{Ngā Tuhinga Tawhito}

MS-0001, Edward Shortland Papers, Te Uare Taoka o Hākena, Te Whare Wānanga o Ōtākou.

MS-0002, Edward Shortland Papers, Te Uare Taoka o Hākena, Te Whare Wānanga o Ōtākou. 